\title{
CFD/CSD trim coupled simulation of the HART II rotor with higher harmonic control
}

\author{
Projektgruppe/Fachkreis: Drehflügleraerodynamik \\ Author: \\ Institute: \\ Annika Länger \\ e-mail: \\ DLR Institute of Aerodynamics and Flow Technology \\ annika.laenger@dlr.de \\ Phone: 0531-2952439 \\ 38108 Braunschweig
}

\begin{abstract}
The TAU-HOST coupling chain for Fluid-Structure Interaction (FSI) simulations of active helicopter-rotors is validated by computing the HART II experiment. The result accuracy of the unstructured flow solver TAU is investigated in the case of a rotor which experiences Higher Harmonic Controls (HHC). Investigations are performed by a code-to-code comparison with the experiment, FLOWer-HOST, and literature. Moreover, a time-step study and mesh refinement study were performed but improvement of Blade-Vortex Interaction (BVI) intensity fell short of expectations. Nevertheless, results of rotor controls, blade loads and blade deformation show good agreement with experimental data and are in accordance with literature.
\end{abstract}

\section{Introduction}

Helicopter rotor blades are long slender bodies. Thus, blade deformations are high and have to be taken into account in aerodynamic and dynamics simulation. Deformations depend on the large aerodynamic forces and their variation during the revolution. Hence, the rotor is an aeroelastic problem which requires an iterative computation procedure to balance structural response on the flow solution and vice versa.

Increased helicopter public acceptance in densely populated areas depends on decreasing helicopter noise. One opportunity to reduce noise during take-off and landing is to minimize BVI by active rotors. Active rotor blades are pitched with frequencies higher than $1 /$ rev (HHC) such that blades do not pass the preceding blade's wake. An experiment to investigate the impact of this technique on rotor noise radiation are the so-called HHC Aeroacoustic Rotor Tests (HART [1]) from 1994 and 2001. Nowadays, the experiment is used as validation case for aeroelastic rotor computations throughout the scientific comunity. An overview of recent efforts is presented by Smith et al. [2] who compared different loosely coupled CFD/CSD routines among each other.

The computation of aeroelastic effects on rotor blades by coupling CFD flow solvers with the comprehensive rotor simulation code HOST (Helicopter 
Overall Simulation Tool) [3] was first established by Altmikus [4] in 2004. He used the structured flow solver FLOWer in a loose coupling procedure. Herein, loose coupling describes aeroelastic computation during which data is transferred once per a defined period of several time steps while strong coupling enables data transfer every time step. In 2012, Boisard et al. [5] demonstrated the capability of their structured CFD solver elsA coupled with HOST. As a new aspect, the unstructured flow solver TAU is used for coupling, herein. The methods and modelling approaches of the TAU-HOST coupling chain was presented in detail by Wendisch [6] who validated the coupling chain with the GOAHEAD projekt [7].

The aim of this work is to further validate the TAU-HOST coupling chain by computing two test cases of the HART II experiment and having the aeroelastic blade deformation and rotor trim determined by the coupling chain. During prestudies, presented in section 3.1 and 3.2, it was ensured that the time step size and the background-grid resolution do not influence results quality while effects as e.g. neglecting the fuselage are more important. A code-to-code comparison with FLOWer-HOST is performed to verify the implementation. In all cases, results are compared to experiments and literature.

\section{Numerical methods and computational setup}

\subsection{Numerical methods}

The flow computations were performed with the DLR unstructured solver TAU [8] and the structured flow solver FLOWer [9] that solve the Reynolds Averaged Navier-Stokes (RANS) equations. Turbulence is modelled with the Wilcox $k-\omega$ model. Computations were run on a time-accurate dualtimestepping approach using a central scheme with scalar dissipation. Grid motion is enabled by the chimera technique. Differences occure in the spatial metric as TAU uses a cell-vertex dual grid approach while FLOWer is cellcentered.

HOST [3] was developed by Airbus Helicopters France and is able to determine the rotor trim and blade motions for isolated rotors as well as the entire helicopter in a given flight state. For this, HOST combines structure analysis with 2D aerodynamic models from the lifiting line theory and lookup tables. Extensions to consider wakes and other arodynamic effects are available. HOST modells use linearised finite linear beams with 3 rotational degrees of freedom ([3]) to approximate rotor blades. Blade motions are composed by supperposing the uncoupled eigenmodes of flap, lead-lag, and torsion.

\section{$2.2 \quad$ Loose coupling chain}

Rotor controls define the blade pitch motion and have the form

$$
\Theta=\Theta_{0}+\Theta_{1 c} \cos \Psi+\Theta_{1 s} \sin \Psi+\Theta_{H H C}
$$


wherein $\Theta_{0}$ is the collevtive control, $\Theta_{1 c}$ is called lateral control, $\Theta_{1 s}$ is the longitudinal control, and $\Theta_{H H C}$ defines higher frequency controls. $\Psi$ is the rotor azimuth angle. Higher frequency pitch motions that are applied during the HART II computation have the form

$$
\Theta_{H H C}=\Theta_{3} * \cos \left(3 \cdot \Psi-\Delta \Psi_{3}\right) .
$$

Therein, $\Theta_{3}$ is the amplitude and $\Delta \Psi$ is the phase shift of the HHC motion. The loose coupling chain starts with computing the rotor trim and blade deformations with HOST, using HOST-internal aerodynamic loads. The information is submitted to TAU, which solves the flow equations. After a rotor rotation of $\Delta \Psi=2 * 360^{\circ} / i$, with $i$ beeing the number of rotor blades, the $3 D$ surface loads are integrated to compute the loads along the $q_{1 / 4}$-line of the rotor blade $F_{C F D}(\Psi)$ which are then transmitted to HOST. These aerodynamic loads are then used by HOST to correct the $2 D$ blade loads $F_{2 D}(\Psi)$ according to

$$
F_{H O S T}^{n}(\Psi)=F_{2 D}^{n}(\Psi)+F_{C F D}^{n-1}(\Psi)-F_{2 D}^{n-1}(\Psi)
$$

with $n$ denoting the $n^{\text {th }}$ coupling cycle (Wendisch [6]). Based on the new blade loads, HOST adjusts control angles and blade deformation. The coupling cycle is repeated until the difference between sectional blade loads $F_{2 D}^{n}(\Psi)$ and $F_{2 D}^{n-1}(\Psi)$ is zero and $F_{H O S T}(\Psi)=F_{C F D}(\Psi)$ (Wendisch [6]).

\subsection{Blade geometry and grid topology}

The HART II test case was conducted with a four-bladed model of the Bo105 rotor with a radius of $R=2 \mathrm{~m}$ and a constant chord length of $c=0.121 \mathrm{~m}$. A modified NACA 23012 profile with a truncated trailing edge (tab lenght $l=0.005 \mathrm{~m}$ ) was used between the radial positions of $0.22 R \leq r \leq R$. The blade is constantly twisted with $\Theta(r)=-8^{\circ} \mathrm{r} / R$ and the zero twist angle is at $r_{0}=1.5 \mathrm{~m}$.

The chimera grid setup consists of 4 identical block-structured child grids with approximately 1.2 Mio. points each, and a background grid with equally spaced elements in the rotor plane and an unstructured grid in the farfield. The volume between the structured area to the farfield boundaries is filled with unstructured grid. An overview of the setup is displayed in figure 1 and a slice through the child mesh ist displayed in figure 2 . Two variants, a coarse (A) and a fine grid (B), with a grid spacing of $\delta_{A}=0.2 c$ and $\delta_{B}=0.1 c$ in the structured background grid region are generated. The background grid A containes approximately 9.7 Mio. points, while background grid B consists of approximately 67.4 Mio points.

To perform a code-to-code validation with FLOWer, a FLOWer background grid was generated, based on grid A. Grid spacing in the rotor plane region was reduced to $\delta \cong 0.15 c$. The unstructured region was replaced with blockstructured mesh. 


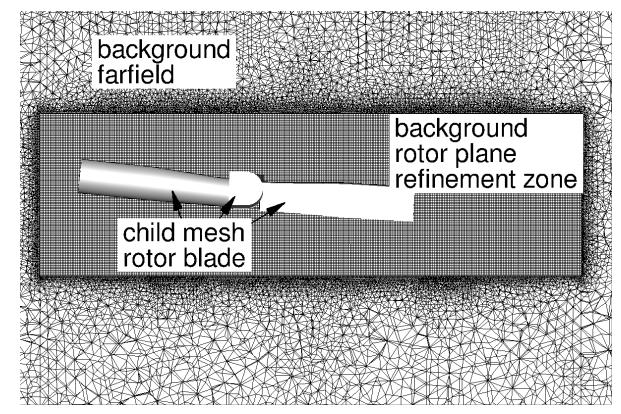

Figure 1: Grid setup

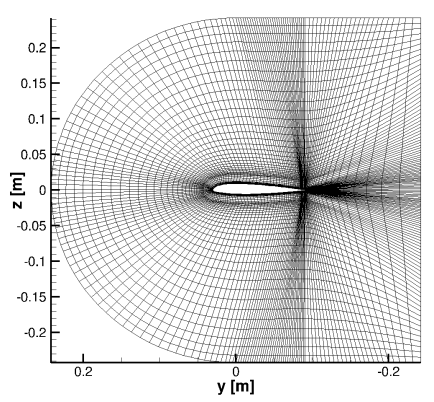

Figure 2: Chimera child grid

\subsection{Test case}

The computed isolated rotor is in descending flight with an advance ratio of $\mu=0.151$. Wind tunnel corections are applied to the installed rotor shaft angle $\alpha_{\text {shaft }}=-5.3^{\circ}$ such that the effective angle of attack is $\alpha_{\text {eff }}=-4.5^{\circ}$ [1]. In this flight conditions, the rotor experiences strong BVI. The hover tip Mach number is $M_{t}=0.693$ at a rotational speed of $109 \mathrm{rad} / \mathrm{s}$. Air is close to standard atmosphere at height $H=0 \mathrm{~m}$ ([1]). The rotor is trimmed to produce a thrust of $T=3 \mathrm{kN}$.

Two test cases were defined during the HART II experiment: The baseline case (BL) describes the case without HHC. It defines the controls, noise and blade load references. The minimum noise (MN) case describes the minimized rotor noise level. It is reached with $\Theta_{3}=0.8^{\circ}$ and a phase of $\Delta \Psi_{3}=300^{\circ}$. Following the argumentation of Smith et. al [1], mean values are removed from all aerodynamic blade loads.

\section{Prestudy - Baseline case}

\subsection{Grid refinement study}

In the grid refinement study the BL case was computed on grid A and B, respectively. By comparing rotor controls and blade loads, the impact of the grid resolution is quantified.

By evaluating the blade loads at the position $r=0.87 R$ (figure 3 ) it has been found, that BVI effects are computed differently, which can be seen on both advancing and retreating side. In grid A (figure 5(a)), only the vortex of the preceeding blade is kept whereas on grid B (figure 5(b)), vortices are kept about $\Delta \Psi=270^{\circ}$ in the second to fourth quadrant. Thus, with the fine background grid BVI-induced oscillating forces between $270^{\circ} \leq \Psi \leq 360^{\circ}$ are experienced by the blades. The four predicted BVI peaks detected in figure 3 are documented in the experiment even though, amplitudes and phase of computation and experiment do not match. As the blade grid is not refined 


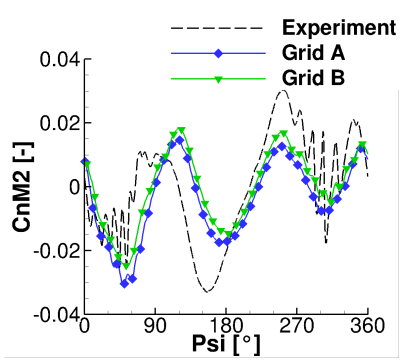

Figure 3: Blade loads in dependence of the grid resolution

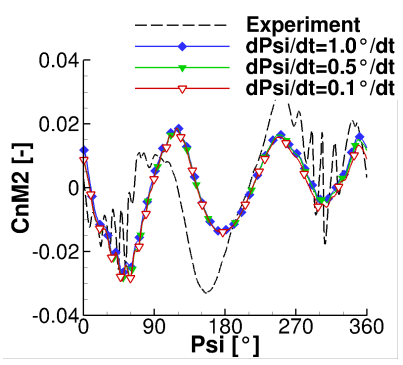

Figure 4: Blade loads in dependence of the time step sizes

in this study, the impact of higher grid density in that part of the grid has to be investigated in further computations. Contrarly, the BVI-like structures on grid A (figure $5(\mathrm{a})$ ) around $\Delta \Psi=60^{\circ}$ are caused by three vortex fingers which result from numerical inaccuracies in the chimera overlapping region rahter than physics. As their strength decreases with refined meshes, the BVI-like oscillations vanish of the blade loads embedded in grid B.

Rotor controls are slightly different between both computations (see table 1 ). $\Theta_{0}$ and $\Theta_{1 c}$ increased about $0.2^{\circ}$ while the longitudinal control angle $\Theta_{1 s}$ decreased about $0.1^{\circ}$ and is closer to experiment. Due to the small impact of backtround-grid refinement grid $\mathrm{A}$ is chosen in the following studies.

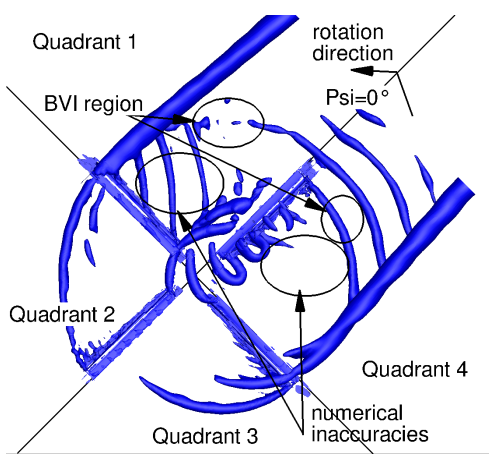

(a) Field solution, grid A

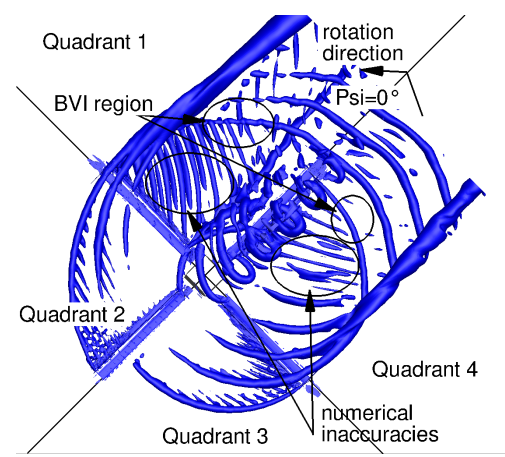

(b) Field solution, grid B

Figure 5: Vortex trajectories in dependency of the grid; $\lambda_{2}$-criterion, top-view

\subsection{Time step study}

In this study, the dependence of the flow solution on the physical time step $\delta t$ is investigated. Thus, the periodic solution of azimuthal steps with $\Delta \Psi=$ $1.0^{\circ} / \delta t, \Delta \Psi=0.5^{\circ} / \delta t$ and $\Delta \Psi=0.1^{\circ} / \delta t$ are compared. Figure 4 contains the blade loads of the initial trim of all three time steps at $r=0.87 R$. It is distinguishable, that with the largest azimuthal step global flow phenomena 
Table 1: Converged control angles of the prestudies, all in $\left[^{\circ}\right]$

\begin{tabular}{|l|l|l|l|l|}
\hline & grid A & grid A & grid B & $\begin{array}{l}\text { Experi- } \\
\text { ment }\end{array}$ \\
& $d \Psi / d t=1.0^{\circ} / d t$ & $d \Psi / d t=0.5^{\circ} / d t$ & $d \Psi / d t=1.0^{\circ} / d t$ & 3.8 \\
$\Psi_{0}$ & 3.49 & 3.49 & 3.51 & 1.92 \\
$\Psi_{1 c}$ & 1.68 & 1.68 & 1.70 & -1.34 \\
$\Psi_{1 s}$ & -0.34 & -0.35 & -0.35 & \\
\hline
\end{tabular}

are sufficiently resolved. Rotor advances smaller than $\Delta \Psi=0.5^{\circ} / \delta t$ lead to completely time step independent solutions as BVI are identical for $\Delta \Psi=$ $0.5^{\circ} / \delta t$ and $\Delta \Psi=0.1^{\circ} / \delta t$.

Out of figure 4 it was not expected, that control angles change much because they depend on the global flow solution. Indeed, converged collective control $\Theta_{0}=3.49^{\circ}$ is not changed whereas lateral and longitudinal controls are reduced about $0.1^{\circ}$ respectively (table 1 ). Thus, the time step size of $\Delta \Psi=$ $1.0^{\circ}$ is chosen for validation of the TAU-HOST coupling chain to active rotors.

\section{Results}

\subsection{Baseline case - FLOWer-HOST comparison}

To prove that TAU-HOST solutions are equivalent to FLOWer-HOST solutions, a code-to-code comparison for the BL case was performed, regarding control angles, blade loads and deformations. During the study, identic chimera child meshes were used but differing background grids (section 2.3). Comparisons of disk plots of FLOWer-HOST and TAU-HOST in the initial trim are displayed in figure 6 wherein the drag forces $\left(F_{x}, F_{y}\right)$ and the lift force $\left(F_{z}\right)$ are plotted in the upper line. Flow is coming from the left side and the rotor is rotating anti-clockwise. In the lower row of figure 6 , difference plots between TAU and FLOWer are displayed. Differences are small and lie between $-5 \mathrm{~N} \leq F_{x} \leq 5 \mathrm{~N}$ and $-2 \mathrm{~N} \leq F_{y} \leq 2 \mathrm{~N}$ for the drag forces. In the difference plot of $F_{z}$ and $F_{y}$, it can be seen, that TAU captures the vortices different than FLOWer as the vortex structure is visible in both plots. This is due to different point positions in the cell-centered and cell-vertex schemes and differences in the background grid density.

Control angle convergence was evaluated via the relative change from one coupling cycle to the other. Convergence is reached if the HOST adjustment is less than $1 \%$. Figure 7 displays the convergence history. Convergence is reached after 8 coupling cycles. During the entire computation, rotor controls differed less than $0.2^{\circ}$ between FLOWer-HOST and TAU-HOST. Converged controls are listed in table 2 and differ from experiment about $\Delta \Theta_{0}=\left(\Theta_{0}-\Theta_{0_{e x p}}\right) / \Theta_{0_{e x p}}=-9.5 \%, \Delta \Theta_{1 c}=13.02 \%$ and $\Delta \Theta_{1 s}=57.5 \%$ due to the missing fuselage. While $\Theta_{0}$ and $\Theta_{1 c}$ match the experiment and 

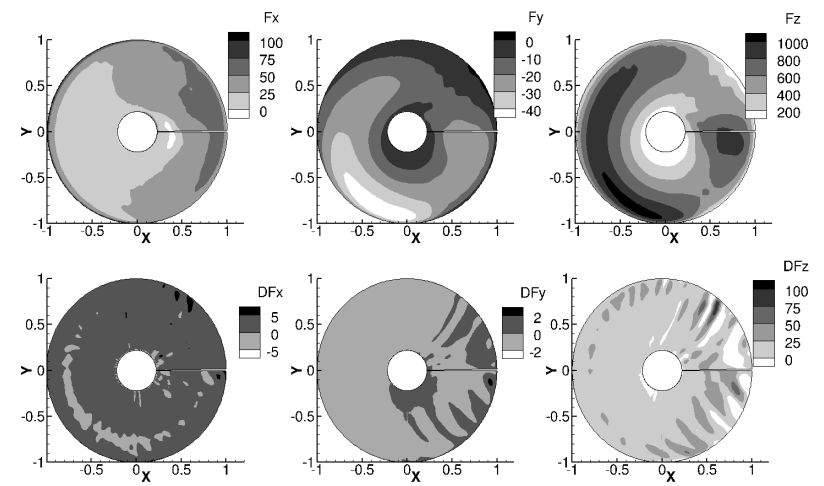

Figure 6: Blade loads in TAU (upper) and differences to FLOWer (lower)

reach the level of comparable studies in literature (e.g. [5]), $\Theta_{1 s}$ shows slightly larger discrepancies.

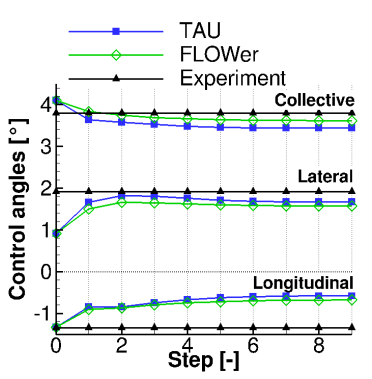

Figure 7: Control angle convergence

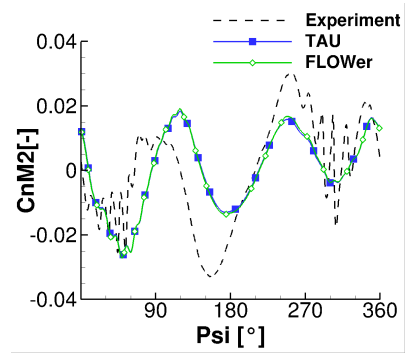

Figure 8: Aerodynamic blade loads, TAU-HOST vs.

FLOWer-HOST and experiment

In figure 8 the aerodynamic loads extracted at $r=0.87 R$ of the BL case of both flow solvers are compared to the experiment. Apparently, FLOWer and TAU blade loads differ in BVI region only. Compared to experiment, the blade experiences too high forces in the range of $90^{\circ} \leq \Psi=180^{\circ}$ and to small forces at $180^{\circ} \leq \Psi \leq 270^{\circ}$ which results from the missing fuselage and the blade root vortices that are caused by neglecting the rotor head, respectively. The phase offset between experiment and computations at $90^{\circ} \leq \Psi \leq 270^{\circ}$ results from the isolated rotor setup, whose impact has been investigated by Park [10]. After passing $\Psi=270^{\circ}$, global flow solution matches the experiment well. All in all, the characteristics of the measurements from HART II experiment were met well by the computation.

Following the argumentation of Smith et al. [2], tip deformation is only 
correct if deformation along the entire blade is computed correctly, solely blade tip deflections are discussed herein. During the experiment it was noticed, that all 4 blades have different stiffness properties and thus motion differs significantly from one blade to the other [1]. Thus, computational results are compared to those two blades which mark the upper and lower boundary of measured blade motion. Regarding code-to-code differences, displayed throughout figure 9 , it is found that blade flap and lead-lag motions of TAU-HOST and FLOWer-HOST coincide almost perfectly while torsion motions are very close. Compared to experimental measurements, computed flap motions (figure 9(a)) lie in the spread of blade-to-blade differences between $90^{\circ} \leq \Psi \leq 270^{\circ}$. In the rear rotor part $\left(90^{\circ} \geq \Psi ; 270^{\circ} \geq \Psi\right)$, blade flap deformation shows significant discrepancies. Similar behaviour was detected by Boisard et al. [5]. He also found similar deviations in blade torsion motion which significantly deviate from experiment around $\Psi=80^{\circ}$ in TAUHOST computation. Boisard related the motion discrepancies to the large discrepancy in longitudinal control and stats that, the longitudinal control and the torsion motion compensate each other. Superposing blade ptich motion and elastic torsion lead to correct overall blade motion [5]. As blade torsion is measured as difference of the chord line angle between rotating and non-rotating blade, slight differences in the chord-line definition lead to a constant offset between experiment and computation.

As proposed throughout literature $[2,5,10]$, only lead-lag motion around the mean values are considered, as the offset from experiment to computation is large. Computed and measured lead-lag deformation around the mean value fit the experiment very well (figure 9(c)).

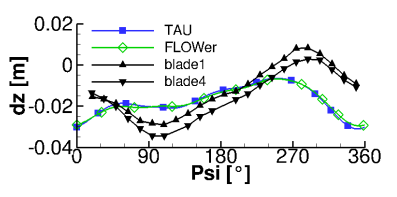

(a) Flap

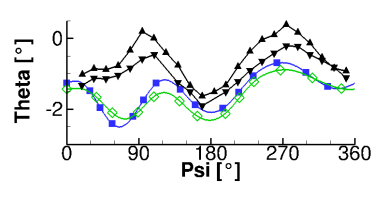

(b) Elastic torsion

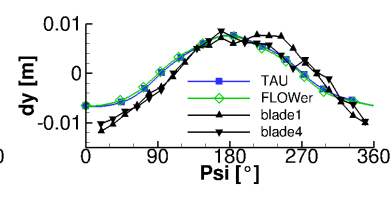

(c) Lead-lag

Figure 9: Comparison of blade tip deformation

Table 2: Comparison of control angles, all in $\left[^{\circ}\right]$

\begin{tabular}{|l|l|l|l|l|l|}
\hline & Experiment & FLOWer-HOST & TAU-HOST & Experiment & TAU-HOST \\
& BL & BL & BL & MN & MN \\
\hline$\Theta_{0}$ & 3.8 & 3.61 & 3.44 & 3.9 & 3.48 \\
$\Theta_{1 c}$ & 1.92 & 1.58 & 1.67 & 2.0 & 1.69 \\
$\Theta_{1 s}$ & -1.34 & -0.66 & -0.57 & -1.35 & -0.42 \\
\hline
\end{tabular}




\subsection{Minimum noise case}

MN controls are determined to $\Theta_{0}=3.48, \Theta_{1 c}=1.69$, and $\Theta_{1 s}=0.42$. Thus, the adjustment of controls in computation is less than expected from experimental results (table 2). Nevertheless, $\Theta_{0}$ and $\Theta_{1 c}$ are in the range of comparable studies ([2]) while the longitudinal controls are unexpectedly large. Reasons are identic to the BL case.

Aerodynamic blade loads (figure 10) of the MN case clearly show the $3 /$ rev characteristics. Moreover, global force amplitudes and phase is in very good agreement with experiment during the entire revolution. BVI oscillations at the advancing rotor side are due to numerical inaccuracies while vortices are not maintained long enough to induce BVI oscillations around $\Psi=300^{\circ}$ (section 3.1).

As expected, the $3 /$ rev characteristics, induced by the HHC, has an impact on the flap and torsion motion, while the lead-lag

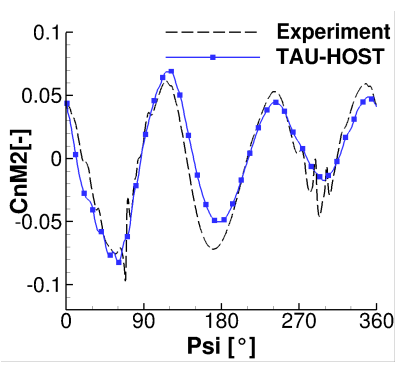

Figure 10: Aerodynamic blade loads, MN case motion is hardly affected (figure 11(c)).

As has been seen at the BL case, especially the blade torsion motion is computed excellent during the entire rotation by TAU-HOST (figure 11(b)). Moreover, the lead-lag deformation coincide well with the experiment. In contrast, flap amplitudes are underpredicted at the advancing rotor side but better reproduced at the retreating rotor side. This behaviour has already been seen in the BL case and by Boisard et al. [5] and thus is assumed to result from the simplifications in the HOST model.

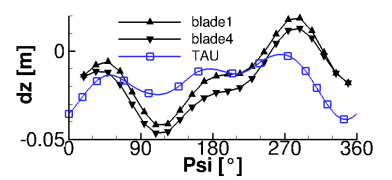

(a) Flap

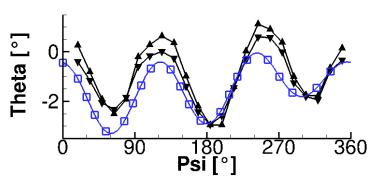

(b) Elastic torsion

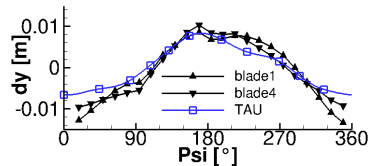

(c) Lead-lag

Figure 11: Comparison of blade tip deformation

\section{Conclusion}

The present work demonstrats the capabilities of the TAU-HOST coupling chain to compute active rotors whith HHC. Considering the numerical setup, results of the BL and MN case are good. Collective and lateral control angles between computation and experiment differed about $-0.4^{\circ}$ and $0.9^{\circ}$ for the 
longitudinal control. Global flow phenomena and blade motion characteristics were well reproduced. However, to improve results in future work the fuselage has to be added to the numerical setup. If efforts will be made, to better capture BVI effects, higher order schemes in the flow solver, finer blade grids, and time step sizes are needed. Additionally, a more complex model for structure analysis has to be used.

\section{References}

[1] B.v.d.Wall et al. The HART II test in the LLF of the DNW - a major step towards rotor wake understanding. $28^{\text {th }}$ European Rotorcraft Forum Proceedings, September 2002.

[2] M. Smith et al. An assessment of CFD/CSD prediction state-of-the-art using the HART II international workshop data. $68^{\text {th }}$ AHS annual forum, May 1-3,2012.

[3] B. Benoit et al. HOST, a general helicopter simulation tool for germany and france. $56^{\text {th }}$ AHS annual forum, May 2-4,2000.

[4] A. Altmikus. Nichtlineare Simulation der Strömungs-StrukturWechselwirkung am Hubschrauberrotor. PhD thesis, Institut für Aerodynamik und Gasdynamik, University Stuttgart, 2004.

[5] R. Boisard et al. Assessment of aeromechanics and acoustics methods for bvi prediction using CFD. $39^{\text {th }}$ European Rotorcraft Forum, September 2012 .

[6] J.-H. Wendisch;J. Raddatz. Validation of an unstructured CFD solver for complete helicopter configurations with loose CSD-trim coupling. $40^{\text {th }}$ European Rotorcraft Forum, September 1-3,2014.

[7] T. Schwarz; K. Pahlke. Generation of an advanced helicopter experimental aerodynamic data base for CFD validation - The European GOAHEAD project. Aerospace Science and Technology, May 2012.

[8] D. Schwamborn et al. The DLR TAU-Code: recent applications in research and industry. ECCOMAS CDF 2006, September 5-8, 2006.

[9] J. Raddatz; J. Fassbender. Block structured Navier-Stokes solver FLOWer. MEGAFLOW - numerical flow simulation for aircraft design. Notes on Numerical Fluid Mechanics and Multidisciplinary Design, Vol. $89,2005$.

[10] J.-S.Park et al. Loosely-coupled multibody dynamics/CFD analysis for a rotor in descending flight. Aerospace Science and Technology, Vol. 29(1), 2013. 